\title{
Research on Direct Evaporative Solar Ejection Refrigeration System
}

\author{
Liu Wei ${ }^{1, a}$, Liu Liang ${ }^{2, b}$, Chai Huilai ${ }^{3, c}$ \\ ${ }^{1}$ Energy Resources Research Dept., Hebei Academy of Sciences, Shijiazhuang, Hebei, China. \\ ${ }^{2}$ Head Office, NORENDAR International, 55 Yuhua Donglu, Shijiazhuang, Hebei, China. \\ ${ }^{3}$ Hebei University of Engineering, 199 Guangming South Street, Handan, Hebei, China.

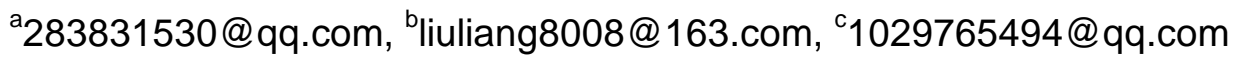

\begin{abstract}
Key words: ejection refrigeration, solar collecting generator, direct evaporation
Abstract: At present, the solar ejection refrigeration system's solar collecting system mainly adopts water as medium for solar energy absorption and then as the heat source of ejection system, which leads to a result that the system must be provided with components like water tank, and circulation pump, etc., and thus the system is of large size with low economical efficiency and its COP (coefficient of performance) is low. To solve this problem, the paper proposes a new kind of solar ejection refrigeration system. Without generator of traditional ejection refrigeration system and adopting integration technology of solar collector and (steam) generator, refrigerant absorbs heat and will directly be evaporated for phase-change heat transfer, which omits the intermediate link of heat exchange and improves the system's cycle performance and energy saving effect.
\end{abstract}

\section{Introduction}

The present solar collecting subsystem mainly uses water as medium for solar energy absorption and then as the heat source of ejection system, which leads to a result that the system must be provided with components like water tank, and circulation pump, etc., and thus the system is of large size with low economical efficiency; moreover, the energy transfer between water and refrigerant increases the system's heat transfer loss, and then reduces the refrigeration cycle's COP (coefficient of performance) of the whole refrigeration system.

Considering the problem of low cycle performance coefficient and economical efficiency of the solar ejection refrigeration system, this project puts forward a new kind of solar ejection refrigeration system -- direct evaporative solar ejection refrigeration system, which omits generator in traditional ejection refrigeration system, adopts integration technology of solar collector and (steam) generator, thus the refrigerant in generator absorbs heat and is directly evaporated for phase-change heat transfer and intermediate links of heat exchange can be reduced. While an in-depth theoretical research is carried out for the core components (ejector) of the ejection refrigeration system to perfect the design of ejection and improve the working efficiency of ejection refrigeration system; hence the cycle performance and energy saving effect of the system can be improved to create a new path for application of solar ejection refrigeration system in the building construction.

\section{Working Principle}

Traditional solar ejection refrigeration system mainly consists of two parts, of which, one is the solar collecting subsystem, the energy source of the system; the other one is ejection refrigeration subsystem, which provides cold production for users. In the solar thermal conversion subsystem, the solar collector converts solar energy into heat, and then transfers it to the generator to heat the liquid working medium in it so as to make it evaporate. In cooling sub-cycle, the liquid refrigerant absorbs heat to generate saturated steam, passes through the ejector, and then generate low pressure near the nozzle in the ejector receiving chamber so as to suck the refrigerant steam from evaporator into ejector's mixing chamber, mix it in the chamber, and increase pressure at the ejector's diffusion section; 
the mixed refrigerant gas from ejector enters into condenser and condensation will be provided, liquid refrigerant from condenser enters into the fluid reservoir and then is divided into two lines, of which, one line will return to the evaporator after pressure reduction by passing through the throttle valve, the other line will enter into the generator after boosted by circulation pump, thus the whole refrigeration cycle process is completed.

The present solar collecting system mainly uses water as medium for solar energy absorption and then as the heat source of ejection system, which leads to a result that the system must be provided with components like water tank, and circulation pump, etc., and thus the system is of large size with low economical efficiency; moreover, the energy transfer between water and refrigerant increases the system's heat transfer loss, and then reduces the refrigeration cycle's COP of the whole ejection refrigeration system.

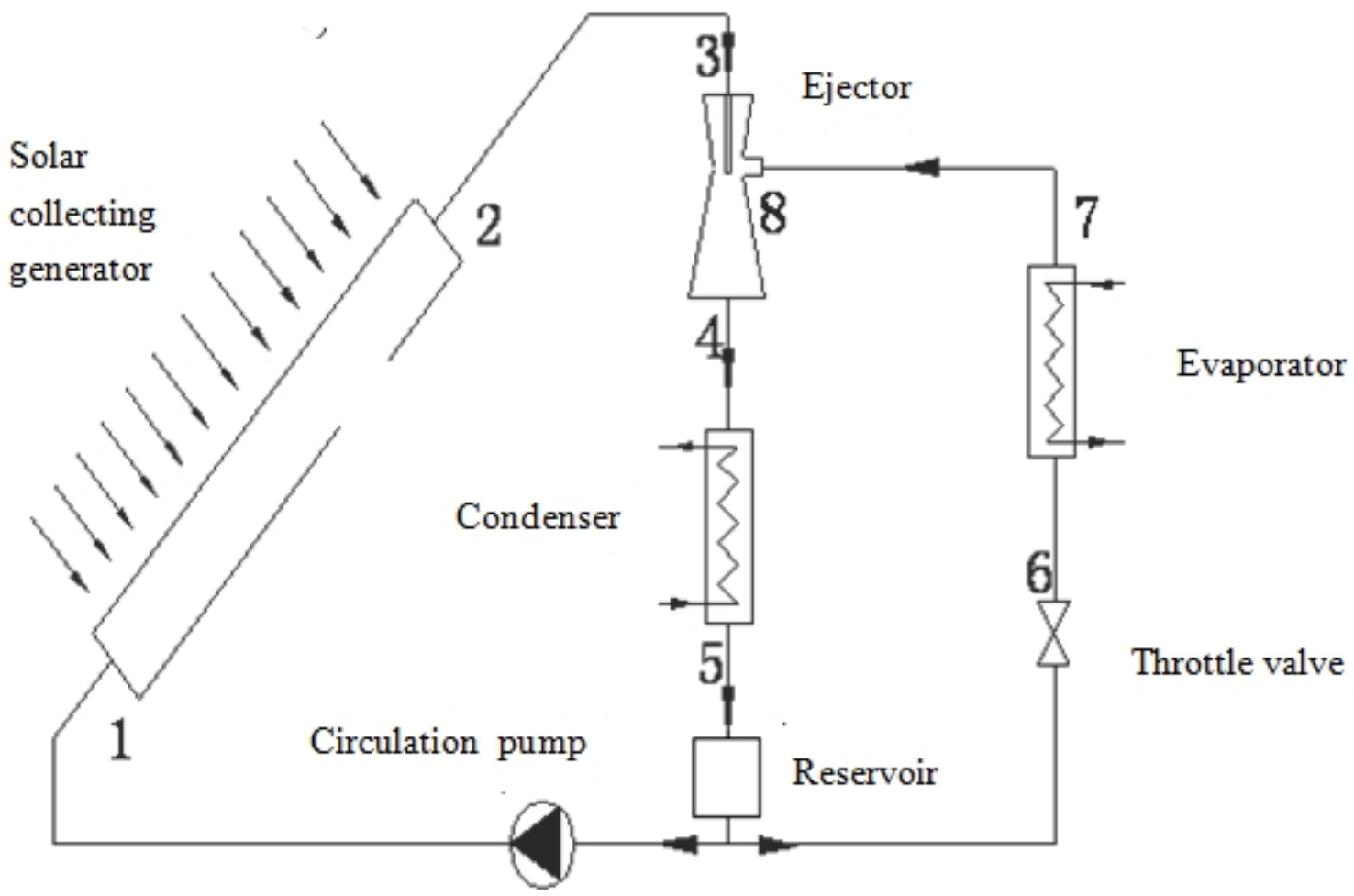

Fig.1 Schematic Diagram of Solar Ejection Refrigeration System

\section{Experimental System}

\section{Numerical simulation}

The two fluids commonly used in FLUENT software, Fluid and Solid, were available in database; and fluid physical parameters could directly called from the database conveniently, such as water, air, R11 and so on. However, refrigerant R134a selected for the project was not in the database. Its input could be performed through Materials panel or calling UDF function method, and the boundary conditions here denoted temperature, pressure and other variables on the boundary of computation domain. In order to obtain the solution of flow field in FLUENT software, reasonable boundary conditions must be determined at first, and the boundary conditions were set as below:

(l) Inlet boundary conditions: For compressible fluid, velocity inlet boundary condition couldn't be selected, thus the inlet boundary of this project used fluid inlet pressure boundary conditions; inlet pressure condition was good in convergence; and turbulence parameters were defined as well. 
(2) Outlet boundary conditions: Fluid outlet pressure boundary condition was selected and static pressure here was given.

(3) Axis boundary condition: The project established a two-dimensional axis symmetric model, whose symmetry axis were zero in velocity component; at the same time, at the direction of symmetry axis, all variable gradients were of zero, thus there was no need to solve the whole computational domain and workload would be greatly reduced.

(4) Near-wall boundary conditions: No-slip adiabatic wall was adopted.

\section{Temperature field distribution in ejector}

The working fluid changed into high-temperature and high-pressure refrigerant steam after heated by collecting generator; after passing through the ejector's nozzle, the steam's speed increased and resulted a larger steam kinetic energy, and its inner energy reduced and temperature dropped rapidly according to the law of conservation of energy, Then after fully mixing in cylindrical section of the ejector's mixing chamber, it became stable. When the mixed fluid flowed through the ejector's diffuser, its speed and kinetic energy gradually decreased, while the temperature gradually increased. The temperature field distribution in the ejector is shown in Figure 2.

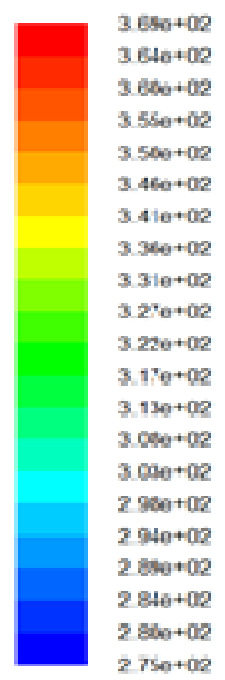

Fig.2 Temperature Field Distribution in Ejector

\section{Velocity field distribution in ejector}

The speed of working fluid flowing through nozzle increased gradually and reached supersonic speed when the fluid passing through nozzle outlet and entering receiving chamber. Then the driving fluid was sucked into mixing chamber and the mixed boundary layer was formed. At this moment, shock wave could easily occur at the nozzle outlet section and the diffusion chamber inlet section. In this process momentum transfer occurred between working fluid and driving fluid, resulting in decreasing speed of working fluid and increasing speed of driving fluid; two fluids tended to be stable at the section of mixing chamber, and the speed continued to decrease after entering into the ejector's diffuser., The distribution and variation of velocity field in the injector is shown in Figure 3. 


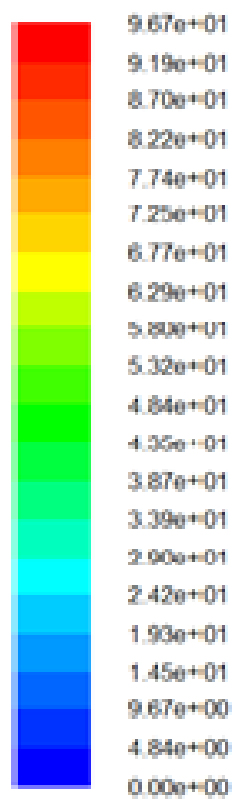

Fig.3 Velocity Field Distribution in Ejector

\section{Pressure field distribution in ejector}

Before and after passing through the ejector nozzle, the pressure of working fluid changed dramatically; the pressure dropped sharply after the Laval nozzle and became lower than that of driving fluid, which would be sucked into the ejector's receiving room. And that was the working principle of nozzle. After being mixed in the mixing room in ejector, working fluid and driving fluid tended to be stable, and then the pressure began to increase through the diffuser. Pressure field distribution and changes are shown in Figure 4 and 5.

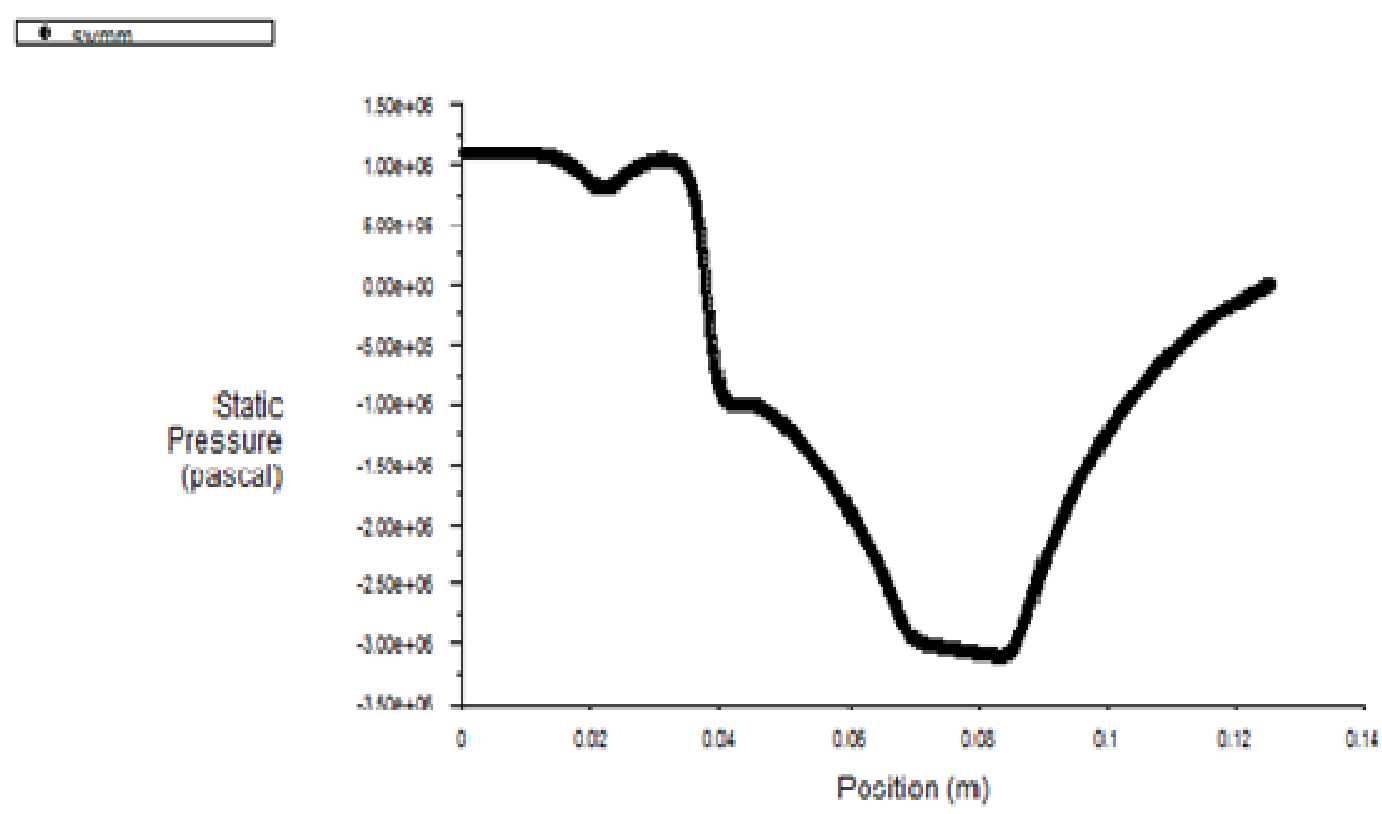

Fig.4 Pressure Change Curve along Axis

From the figure, it can be known that the working fluid surrounding driving fluid at the outlet of ejector nozzle had a higher pressure gradient, which required higher grid fineness. 

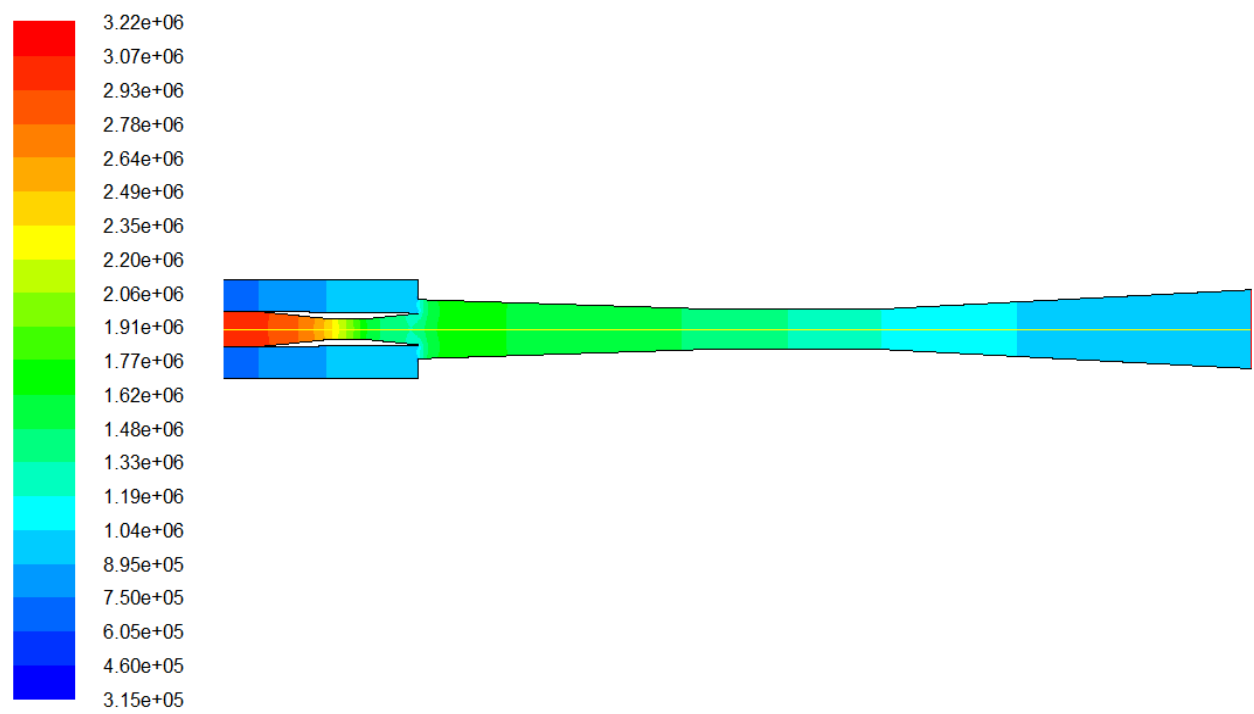

Fig.5 Pressure Field Distribution in Ejector

From the figure, it can be seen that the pressure of working fluid at nozzle outlet was not equal to that of driving fluid but was variable around the driving fluid, so the assumption that the working fluid and driving fluid in ejector are mixed at the same pressure is not accurate. At the same time, the flow field pressure gradient at the nozzle throat and nozzle outlet was relatively larger, and the model grids should be finely divided.

\section{Effect of working fluid pressure on ejector performance}

Under condition that the driving fluid and the mixed fluid at outlet shared the same pressure, simulate and calculate the ejection coefficient of ejector when pressure of working fluid varied from 3.14MPa to $3.26 \mathrm{MPa}$. In the paper, design condition was simulated, i.e. when driving fluid pressure and ejector outlet pressure were $\mathrm{P}_{\mathrm{H}}=0.32 \mathrm{Mpa}, \mathrm{P}_{\mathrm{C}}=0.92 \mathrm{MPa}$ respectively, the ejection coefficient varied with the working fluid pressure $\mathrm{P}_{\mathrm{P}}$.

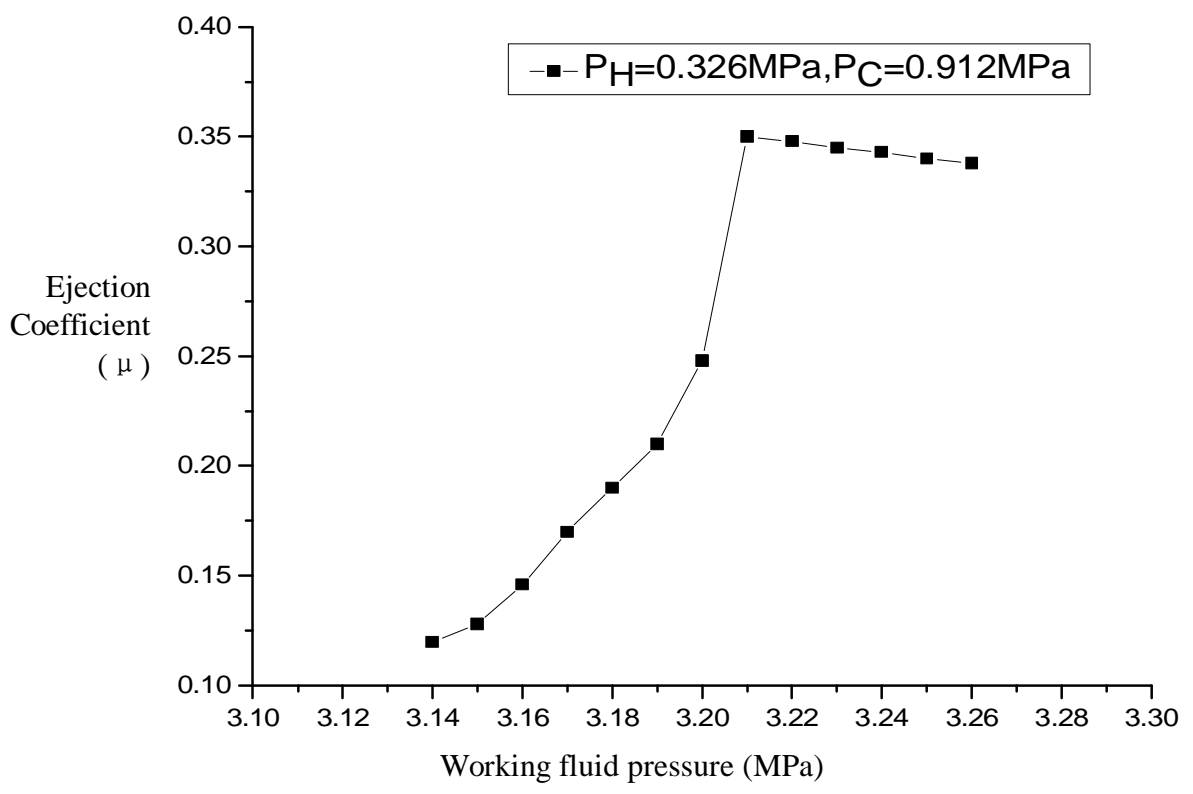

Fig.6 Variation of ejection coefficient with working fluid pressure

From Figure 6 It can be read that when the working pressure $\mathrm{P}_{\mathrm{P}}=3.14 \mathrm{MPa}$, the ejection coefficient was 0.12 , which was the minimum value within the scope of this study section; if the work pressure continued to be reduced, the ejection coefficient would be getting smaller, even became negative. 
Conversely, the ejection coefficient would increase while working pressure increased. Therefore, when $\mathrm{P}_{\mathrm{P}}<3.2 \mathrm{lMPa}$, the ejection coefficient increased with the increasing of the working fluid pressure; when $P_{P}$ reached 3.21MPa, the coefficient reached its peak of 0.35 ; then continue to increase the working fluid pressure, but the coefficient would decrease slightly -- the simulated design condition's ejection coefficient would decrease from 0.34 to 0.30 . Therefore, the ejection coefficient couldn't simply be increased by increasing the working fluid pressure; there was an optimal value of the working pressure, and the working fluid should work around this value as possible while designing ejector.

\section{Experimental System}

The performance test stand of the direct evaporative solar ejection refrigeration system is shown in Figure 7:

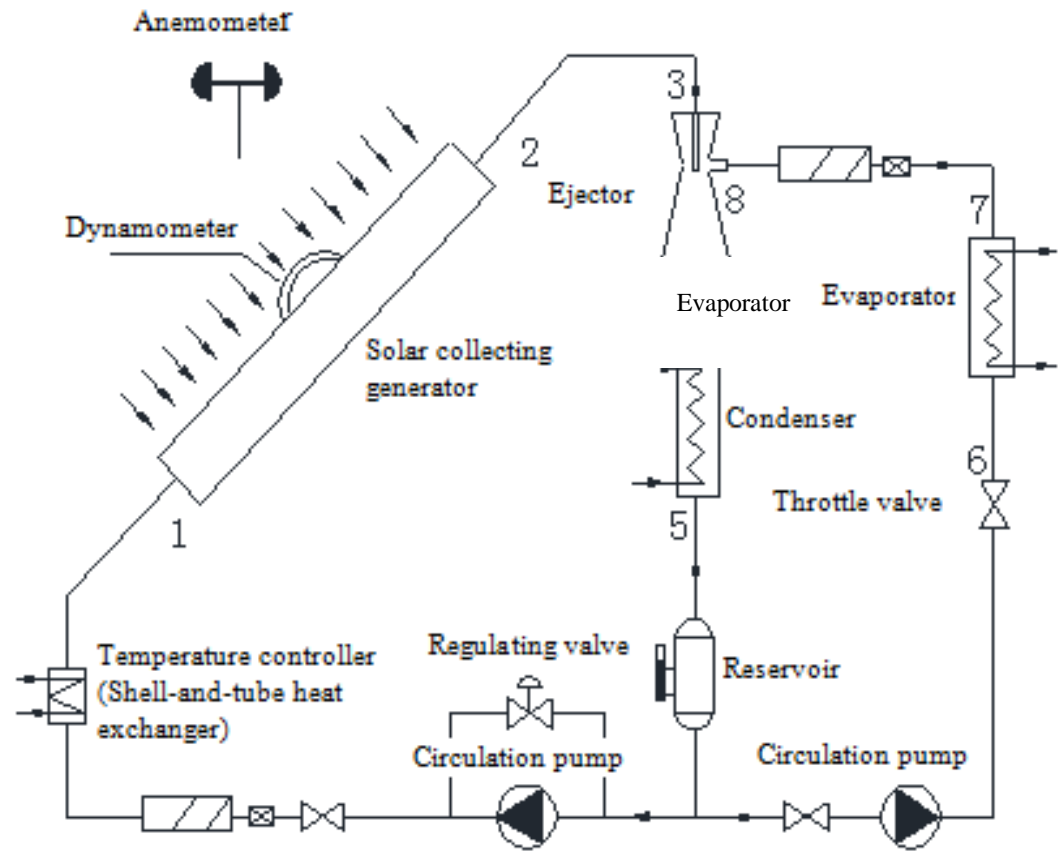

Fig.7 Flow Diagram of Test Stand for Ejection Refrigeration System

The test stand was composed of parts as below:

(1) Direct evaporative plate solar collecting generator unit

The generator unit of designed test stand consisted of coiled copper pipe of $8 \mathrm{~m}^{2}$.

(2) Rotating test stand of direct evaporative plate solar collecting generator

The test device adopted triangle frame from angle steel so as to form a convenient support face for collecting generator in summer; its design angle was $30^{\circ}$ and the surface faced to the sun and tracked it.

(3) Ejector unit for direct evaporative solar ejection refrigeration

The ejector for test had its size designed according to Section 2 and was made of copper.

(4) System circulation pump

This system used R134 refrigerant. In view of refrigerant leakage, the experimental system chose mechanical diaphragm pump, whose model was W100/0.8 - J2M2.

(5) Temperature controller

Self-manufactured shell-and-tube heat exchanger was used; and electric heating water tank was used to control the temperature at the collector inlet.

(6) Condenser

Self-manufactured shell-and-tube water-cooled condenser was adopted.

(7) Evaporator

The evaporator used surface air cooling zone and air quantity was regulated through variable pressure. 
(8) Expansion valve

Manual copper expansion valve was adopted for convenient adjustment in test.

(9) Fluid reservoir

In the designed test, in order to control quantity of the working fluid in control system so as to assure the system's stable running, self-manufactured stainless steel fluid reservoir was used and it was provided with level meter to check the level of fluid in reservoir.

Physical quantities needed to be measured by the designed experimental device mainly were as follows:: system circulation refrigerant flow, flow rate of cooling water, cooling water temperature, solar irradiance, ambient temperature, wind speed, temperature of working fluid at collecting generator inlet and outlet, temperature of working fluid at the condenser inlet and outlet, temperature of working fluid at evaporator inlet and outlet, pressure of working fluid at collecting generator inlet and outlet, pressure of working fluid at the condenser inlet and outlet, pressure of working fluid at evaporator inlet and outlet, and circulating pump power, etc. Moreover, the designed experiment's equipment and connecting pipes were provided with thermal insulation measures. The measuring instruments used in the experiment are shown in Table 1:

Table 1 List of Main Experimental Instruments of the Experimental System

\begin{tabular}{lccc}
\hline \multicolumn{1}{c}{ Description } & Model & Accuracy & Qty \\
\hline $\begin{array}{l}\text { Solar pyranometer } \\
\begin{array}{l}\text { Precision } \\
\text { temperature sensor }\end{array}\end{array}$ & TBQ-2-B & $< \pm 5 \%$ & 1 \\
Ambient & TSD-2A & $\pm 0.1^{\circ} \mathrm{C}$ & 4 \\
temperature sensor & & $\pm 0.1^{\circ} \mathrm{C}$ & 1 \\
$\begin{array}{l}\text { Digital anemometer } \\
\begin{array}{l}\text { Scattered } \\
\text { radiometer }\end{array}\end{array}$ & Tseto 416 & $\pm 0.3 \mathrm{~m} / \mathrm{s}$ & 1 \\
$\begin{array}{l}\text { COX turbine } \\
\text { flow-meter } \\
\text { circulation pump }\end{array}$ & SRT-1 & $< \pm 5 \%$ & 1 \\
\hline
\end{tabular}

\section{Experimental research on performance of ejector}

Preparation was well conducted before the experiment and actual experimental steps were: -

(1) Vacuum pumping of system

Use vacuum pump to create vacuum of the system, and the pressure was less than $0.01 \mathrm{Mpa}$.

(2) Refrigerant filling

After completion of the last step, fill refrigerant into fluid filling port at the lower part of the collecting generator; at the same time, open exhaust valve of fluid filling pipe until there was refrigerant flowing out from the valve. After air in the pipe was discharged completely, fill refrigerant into the collecting generator and then close all valves of the system.

(3) Experiment

According to the experimental requirements, adjust pressure and valve opening to make the system to run stably; and then perform the experimental test data collection. During the whole process of experiment, the working fluid's temperature in generator could also be controlled by the self-manufactured shell-and-tube temperature controller, thus to get the work conditions of ejector under design temperature and other different temperatures. In addition, closely observe the working fluid's temperature and pressure changes at inlets and outlets of the generator, condenser and evaporator during the whole experiment. 


\section{Main Conclusions}

1) Based on numerical calculation of internal flow field of the designed ejector, it showed that: there was a large velocity difference between working fluid and driving fluid at the nozzle outlet, and a discontinuity was formed at the intersection. Under effect of shear force, the driving fluid was accelerated constantly, while on the contrary, the working fluid's speed was decreasing gradually. The momentum and energy exchange between the two fluids happened all the time until there was a balance between them.

2) Through the analysis of plate collector's energy transfer process and calculation of heat transfer coefficient, this paper established the theory model of plate solar collecting generator, thus provided theoretical and data support for collecting generator specially manufactured for experimental test stand.

\section{References:}

[1] T. Solar/waste heat driven two-stage adsorption chiller the prototype [J]. Renew. Energy, 2001, 23:93-101

[2] Viorel Badescu. Model of a space heating system integrating a heat pump, photothermal collectors and solar cells Renewable [J]. Energy, 2002, 27: 489-505

[3] Li Jianhong. Huang Zhicheng. Solar refrigeration and air conditioning research and development in China [J]. Acta Energiae Solaris Sinica, 1999, Special:36-42

[4] Zhang Bo. Research on the Injector and solar ejector refrigeration system [D]. Dalin: Dalian University of Technology, 2002

[5] V.M. Nguyen, S.B. Ri.at, P.S. Doherty. Development of a solar-powered passive ejector cooling system [J]. Applied Thermal Engineering, 2001, 21: 157-168

[6] C.J. Korres, A.T. Papaioannou, V. Lygerou, N.G. Koumoutsos. Solar cooling by thermal compression:The dependence of the jet thermal compressor efficiency on the compression ratio[J]. Energy, 2002, 27:795-805

[7] A.C. Oliveira, C. Afonso, J. Matos, S. Ri.at, M. Nguyen, P. Doherty. A combined heat and power system for buildings driven by solar energy and ga[J]s. Applied Thermal Engineering, 2002, 22:587-593

[8] A.0.Dieng, R..Z. Wang. Literature review on solar adsorption technologies for 1ee-making and air-conditioning purposes and recent developments in solar technology [J]. Renewable \& Sustainable Energy Reviews， 2001，5:313 - 342

[9] Boubakri, Meunier. Adsorptive solar powered ice maker:experiments and mode[J]. Solar. Energy, 2000, 69(3):249-63 